\title{
Boundary Shape and Electrical Impedance
} Tomography

Lionheart, William R.B.

1998

MIMS EPrint: 2008.28

Manchester Institute for Mathematical Sciences

School of Mathematics

The University of Manchester

\footnotetext{
Reports available from: http://eprints.maths.manchester.ac.uk/

And by contacting: The MIMS Secretary

School of Mathematics

The University of Manchester

Manchester, M13 9PL, UK
} 


\title{
Boundary Shape and Electrical Impedance Tomography
}

\author{
W.R.B. Lionheart
}

This is the preprint version of a paper that appeared as

WRB Lionheart, Boundary Shape and Electrical Impedance Tomography, Inverse Problems, Vol 14, No 1, 139-147, 1998

Published version may differ slightly from preprint.

Lionheart is currently at the School of Mathematics, University of Manchester,

Email: bill.lionheart@manchester.ac.uk

Web: http://maths.manchester.ac.uk/ bl 


\title{
Boundary Shape and Electrical Impedance Tomography
}

\author{
W.R.B. Lionheart, \\ School of Computing and Mathematical Sciences, \\ Oxford Brookes University.
}

\begin{abstract}
In Electrical Impedance Tomography (EIT) the boundary shape is often inaccurately known. If the boundary shape is wrong (in a three dimensional problem) there will not generally be an isotropic conductivity which fits the current and voltage data. Both the conductivity and the boundary shape can be determined by electrical data together with three spatial measurements. In two dimensions errors in boundary shape could be accounted for by a change in conductivity, but not if the length scale on the boundary is also known.
\end{abstract}

\section{Introduction.}

In electrical impedance tomography (EIT) one seeks to recover the conductivity from measurements of potential on the surface when a number of current patterns are applied to the surface [1]. This has applications in medical imaging and industrial process monitoring (see for example the conference proceedings [2,3]) In medical applications in particular the body shape is not accurately known. It is thus important to know what influence boundary shape errors might have on reconstructed images. The main result presented here Theorem 5.1 is that if three suitably chosen spatial measurements are made both the conductivity and the boundary shape can be determined by boundary electrical data. This means that in the generic case errors in shape cannot be exactly compensated for by a change in conductivity.

\section{Formulation}

Let the body $\Omega$ be a bounded domain in three dimensional Euclidean space $R^{3}$ with a smooth boundary $\partial \Omega$. Although we are concerned mainly with isotropic conductivity we will have to consider the more general case where the conductivity of the body is given by a symmetric positive definite matrix $\sigma=\left[\sigma_{i j}\right]$ at each point. The potential $\phi$ in the absence of current sources in the interior satisfies

$$
\sum_{i, j=1}^{3} \frac{\partial}{\partial x_{i}}\left(\sigma_{i j} \frac{\partial \phi}{\partial x_{j}}\right)=0
$$

The current density on the boundary is

$j=\sum_{i, j=1}^{3} \sigma_{i j} v_{i} \frac{\partial \phi}{\partial x_{j}}$ 
where $\boldsymbol{v}$ is the outward unit normal to $\partial \Omega$.

We will have need to treat the current on the boundary in a way which is independent of the coordinate system used. To do this we treat current as a differential 2-form J on the boundary. To find the current crossing a given region $R$ of the boundary we evaluate the integral $\int_{R} J$. If $d A$ is the area form on the boundary then $J=j d A$.

The transconductance ${ }^{1}$, or Dirichlet-to-Neumann mapping, $\Lambda_{\sigma}:\left.\phi\right|_{\partial 2}$ a $J$ represents a complete knowledge of the EIT boundary electrical data and the reconstruction problem of EIT is to recover $\sigma$ from $\Lambda_{\sigma}$.

In the anisotropic case, $\sigma$ cannot be determined uniquely from $\Lambda_{\sigma}$. To see this let $F: \Omega \rightarrow \Omega$ be a smooth invertible mapping and define a new conductivity $F_{*} \sigma$ (the push forward of $\sigma$ by $F$ ) by

$$
\left(F_{*} \sigma\right)(x)=\left(\frac{1}{\operatorname{det} D F} D F^{T} \sigma D F\right)\left(F^{-1}(x)\right)
$$

where $D F$ is the matrix of partial derivatives $\left[\partial F_{i} / \partial x_{j}\right]$. The push forward and its inverse, the pull back $F^{*}=\left(F^{-1}\right)_{*}$, are the global equivalents of the transformation rules for the components of a tensor in 'classical' tensor analysis. The conductivity here is a tensor density, hence the determinant in the transformation rule. Provided $F(\mathbf{x})=\mathbf{x}$ for all $\mathbf{x}$ on the boundary we have $\Lambda_{\sigma}=\Lambda_{F_{*} \sigma}[4,5,6]$. At least for analytic conductivities, this is the only reason $\sigma$ is not determined uniquely by $\Lambda_{\sigma}$. That is to say $\Lambda_{\sigma_{1}}=\Lambda_{\sigma_{2}}$ implies $\sigma_{1}=\sigma_{2}$. This result, of Lee and Uhlmann [5] requires the boundary of the domain to be analytic which we will assume is the case. For the two dimensional case Sylvester [6] reduced the anisotropic case to the isotropic case using isothermal coordinates and Nachman [15] proved global uniqueness for less smooth isotropic conductivities.

An anisotropic conductivity determines a Riemannian metric. As a matrix in Cartesian coordinates this is $\left[g^{i j}\right]=(\operatorname{det} \sigma)^{-1}\left[\sigma_{i j}\right]$. Using this metric one can calculate the length and angle between vectors and the length of curves in an electrical sense. As well as the physical geometry we have a non-Euclidean electrical geometry analogous to the way the distribution of matter determines the geometry of space-time in general relativity.

The covariant metric tensor is given by the matrix inverse of the contravariant metric defined above and has components $g_{i j}$ and we define $|g|=\operatorname{det}\left[g_{i j}\right]$. The Laplacian operator with respect to this metric is

\footnotetext{
${ }^{1}$ 'Transconductance' is the natural term for a linear operator taking voltages to currents. 


$$
\Delta_{g}=\sum_{i, j=1}^{3} \frac{1}{\sqrt{|g|}} \frac{\partial}{\partial x_{i}}\left(\sqrt{|g|} g^{i j} \frac{\partial}{\partial x_{j}}\right)
$$

and as $\sigma=\sqrt{|g|} g$ we see that $\Delta_{g} \phi=0$ is equivalent to (1) which is the reason for the introduction of the electrical metric

The electrical inner product of two vectors $\mathbf{X}$ and $\mathbf{Y}$ is $g(\mathbf{X}, \mathbf{Y})=\sum_{i, j=1}^{3} g_{i j} X^{i} Y^{j}$ and the electrical angle between $\mathbf{X}$ and $\mathbf{Y}$ is $\cos ^{-1} \frac{g(\mathbf{X}, \mathbf{Y})}{\sqrt{g(\mathbf{X}, \mathbf{X}) g(\mathbf{Y}, \mathbf{Y})}}$.

Strangely the electrical geometry seems to have only indirect physical relevance. To measure an electrical length and angle one would presumably first have to measure the conductivity.

The push forward of a metric has a simpler transformation formula to that of conductivities:

$\left(F_{*} g\right)_{i j}=\sum_{k, l=1}^{3} g_{k l} \frac{\partial x_{k}}{\partial F_{i}} \frac{\partial x_{l}}{\partial F_{j}}$

A metric e is called flat or Euclidean if its components in some coordinate system are the Kroneker delta $\delta_{i j}$. Two metrics $h$ and $g$ are conformally related if $F_{*} g=\lambda h$ for some smooth invertible map $F$ and positive scalar function $\lambda$. A metric $g$ is called conformally flat if $F_{*} g=\lambda e$ for some smooth invertible map $F$ and positive scalar function $\lambda$. A conformal mapping of a space with metric $g$ is a smooth invertible mapping $F$ with $F_{*} g=\lambda g$ for a positive scalar $\lambda$. A conformal mapping preserves angles while possibly changing lengths.

\section{Unknown Boundary}

If the boundary shape is unknown we can treat the body as an abstract three dimensional manifold $M$ with boundary $\partial M$. A configuration, as in continuum mechanics, is a smooth embedding $\Psi: M \rightarrow R^{3}$ with the body as its image $\Psi(M)=\bar{\Omega}$. A boundary configuration is an embedding $\Psi: \partial M \rightarrow R^{3}$. See Figure 1 .

Suppose we know the transconductance on the abstract manifold. In practical experiments one must attach an array of electrodes to $\partial M$ to apply current and measure voltage. To get the complete transconductance one must be able to make these electrodes arbitrarily small and numerous. To know the transconductance only on the abstract $\partial M$ corresponds to knowing the locations of the electrodes in some coordinate system but not how this coordinate system is embedded in $R^{3}$. We can determine some (possibly anisotropic) conductivity $\sigma$ on $M$ consistent with the measured data and this is defined (by Lee and Uhlmann's theorem) up to some interior distortion $G: M \rightarrow M$, with $G(\mathbf{x})=\mathbf{x}$ for $\mathbf{x} \in \partial M$. Given two possible configurations 
$\Psi_{1}(M)=\bar{\Omega}_{1} \quad$ and $\quad \Psi_{2}(M)=\bar{\Omega}_{2} \quad$ there is a smooth invertible mapping $F=\Psi_{2} G \Psi_{1}^{-1}: \bar{\Omega}_{1} \rightarrow \bar{\Omega}_{2}$. Suppose that we find electrical metrics $g_{1}$ and $g_{2}$ on $\bar{\Omega}_{1}$ and $\bar{\Omega}_{2}$ consistent with our measured data then there must be such an $F$ with $F_{*} g_{1}=g_{2}$.

In the case of an isotropic conductivity the metric $g=\lambda e$ where $e$ is a flat metric and $\lambda$ is a positive function. Such a metric is called conformally flat. The push forward of a conformally flat metric by a smooth invertible map is still conformally flat. We now see that for two configurations as above $F: \bar{\Omega}_{1} \rightarrow \bar{\Omega}_{2}$ is a conformal mapping between domains in Euclidean space, that is $F_{*} e=\lambda e$ for some positive scalar function $\lambda$.

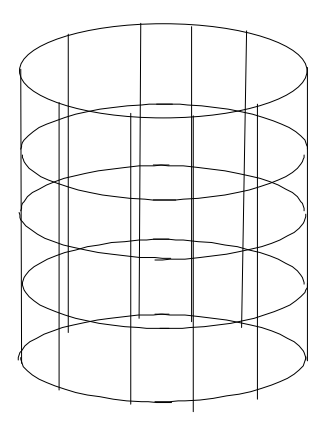

$M$

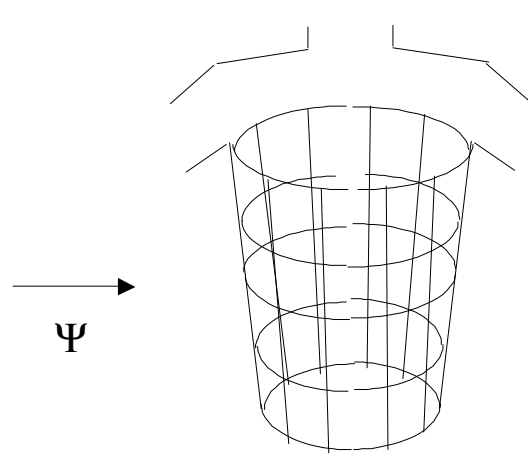

$\Omega$

Figure 1. A portion of the abstract manifold $M$ and a particular configuration, shown here as a chest. A local coordinate system is shown on both boundaries. Electrodes would be placed at known locations in the coordinate system of $M$.

\section{Conformal Mappings}

In the familiar case of a domain in the plane $R^{2}$, conformal mappings correspond to complex analytic functions $f(z)$ on that domain with $\lambda=\left|f^{\prime}(z)\right| \neq 0$. It is easily seen that the space of complex analytic mappings of a domain in the complex plane is infinite dimensional. For domains in higher dimensions the situation is different.

The set of conformal mappings on a given $n$-dimensional manifold $(n \geq 3)$ with a Riemannian metric is a Lie group of dimension no more that $(n+1)(n+2) / 2$ [7]. For example the only conformal mappings on the whole of $R^{3}$ are the similarity transformations: combinations of translations, reflections, rotations and enlargements. Explicitly these are the affine linear mappings $f(\mathbf{x})=\alpha \mathbf{A x}+\mathbf{b}$ where $\alpha$ is a positive scalar, $\mathbf{A}$ is an orthogonal matrix and $\mathbf{b}$ is any vector.

A translation is given by three coordinates, a rotation by three angles and an enlargement by a scale factor. This gives a total of seven parameters needed to specify a conformal mapping of $R^{3}$. Conformal mappings of a subdomain of $R^{3}$ need 
not be the restriction to that domain of a similarity. Indeed they may not be affine linear mappings.

There is a more general class of conformal mappings defined on $R^{3}$ except possibly at one point, these are the Möbius transformations described below. Any conformal mapping defined on a domain in $R^{3}$ can be extended to a Möbius transformation [8]. As we shall see the Möbius transformations can be considered as conformal mappings defined on the whole sphere $S^{n}$ (with its standard geometry inherited from $R^{n+1}$ ). Indeed they constitute the group of all conformal mappings of $S^{n}$ which has the maximum dimension $(n+1)(n+2) / 2$.

If our mapping $F$ could always be extended to the whole of $R^{3}$ the only ambiguity in the boundary configuration would be its position and orientation in space and its overall size. Indeed one would not expect to recover this information from EIT data. The body is connected to the tomograph by flexible wires and the orientation and position of the body should not affect the measurements. A change in the overall size of the body (an enlargement) would have the same effect on the measured data as a scaling of the conductivity. We will say that two subsets of $R^{3}$ have the same shape if they are related by a similarity (that is they are similar in the sense of classical geometry).

There is, however, some further ambiguity due to the Möbius transformations which cannot be extended to the whole of $R^{3}$. To understand these mappings we need to consider the stereographic projection of the sphere $S=\left\{\left(x_{0}, x_{1}, x_{2}, x_{3}\right): \sum_{i=0}^{3} x_{i}=1\right\}$ in $R^{4}$. Denote the point $(1,0,0,0)$ by $\mathbf{n}$ (the 'North Pole') and the (hyper) plane $x_{0}=0$ by $P$. Figure 2 gives a two dimensional illustration of this and the following argument.

A point $\mathbf{x}$ in $P$ is mapped to a unique point $\mathbf{X}$ on $S$ at the intersection with $\mathrm{S}$ of the line through $\mathbf{n}$ and $\mathbf{x}$. This mapping is a smooth invertible mapping $\Pi_{\mathbf{n}}$ from $S-\{\mathbf{n}\}$ to $P$. Moreover it is a conformal mapping between the spherical geometry of $S$ and the Euclidean geometry of $P$ [8]. Now take another 'North Pole' $\mathbf{n}^{\prime}=(0,1,0,0)$ and consider the stereographic projection on to the plane $P^{\prime}$ defined by $x_{1}=0$. Combining this projection with the inverse of the first we have a conformal mapping $F=\Pi_{\mathbf{n}} \Pi_{\mathbf{n}^{\prime}}^{-1}$ from $P^{\prime}-\left\{\Pi_{\mathbf{n}^{\prime}}(\mathbf{n})\right\}$ to $P-\left\{\Pi_{\mathbf{n}}\left(\mathbf{n}^{\prime}\right)\right\}$. One can think of $S$ as representing $P$ with $\mathbf{n}$ as a 'point at infinity' added.

Explicitly we can write $\mathbf{x}=\Pi_{\mathbf{n}}(\mathbf{X})$ in coordinates [8 p95]

$x_{i}=\frac{X_{i}}{1-X_{0}}$, for $1 \leq i \leq 3$

and its inverse $\mathbf{X}=\Pi_{\mathbf{n}}^{-1}(\mathbf{x})$

$X_{0}=\frac{r^{2}-1}{r^{2}+1}, \quad X_{i}=\frac{2 x_{i}}{1+r^{2}}$ for $1 \leq i \leq 3$ 
where $r^{2}=\sum_{i=1}^{3} x_{i}^{2}$. We then have the conformal mapping $F(\mathbf{x})=\Pi_{\mathbf{n}} \Pi_{\mathbf{n}^{\prime}}^{-1}(\mathbf{x})=\frac{1}{1-X_{1}}\left(X_{0}, 0, X_{2}, X_{3}\right)$. 


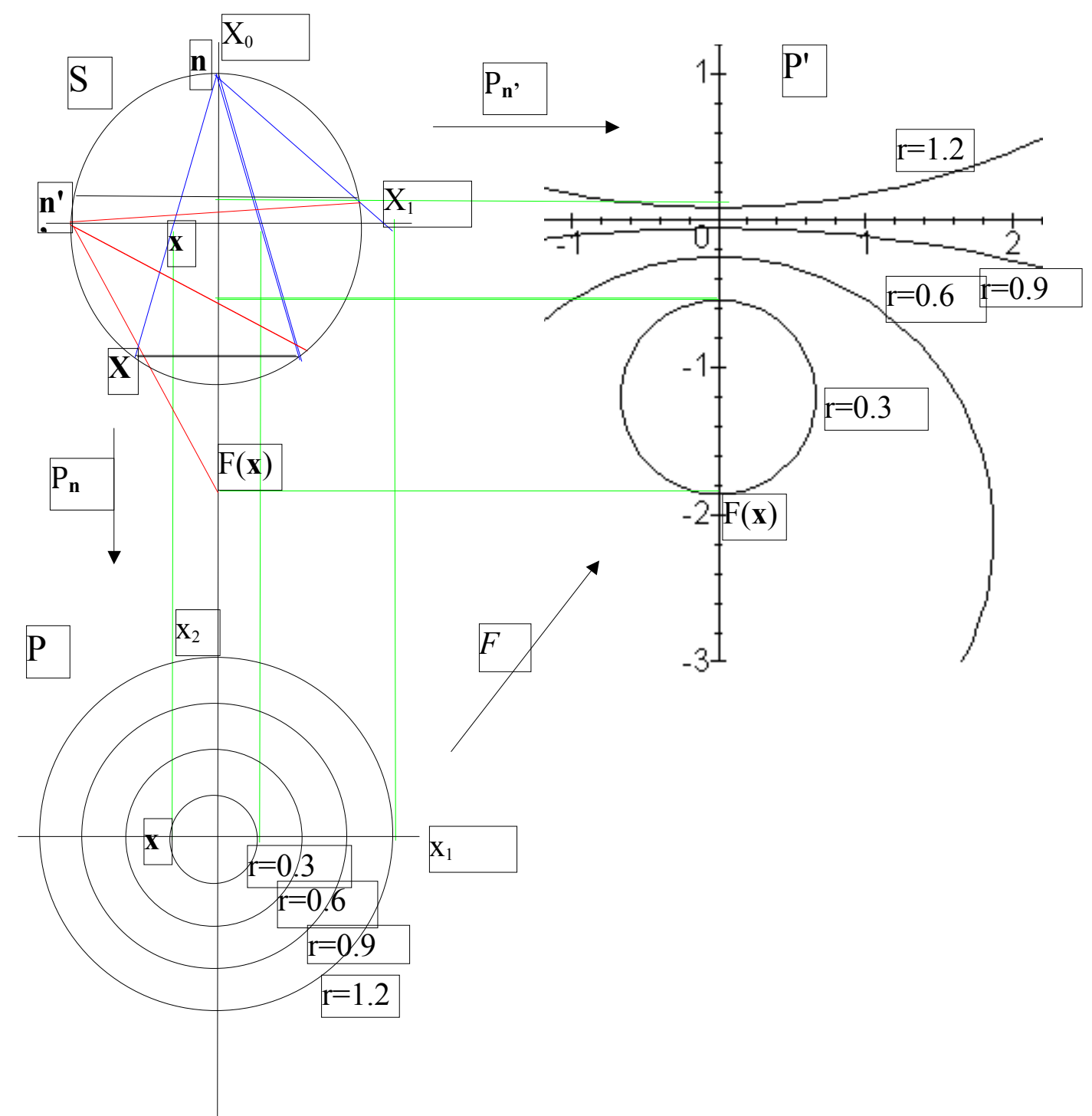

Figure 2. A two dimensional illustration of the Möbius transformation $F$ defined in the text. Starting at the point $\mathbf{x}=(-0.3,0,0)$ on the $P$ plane follow the dotted line upwards to see where this appears on the $X_{0}=0$ plane. The dotted line from $\mathbf{n}$ shows the image $\mathbf{X}$ of this point on $S$. A dotted line from $\mathbf{n}^{\prime}$ through $\mathbf{X}$ intersects the $X_{1}=0$ plane at $F(\mathbf{X})$. Over to the right this is seen on the $P^{\prime}$ plane. The planes $P$ and $P^{\prime}$ have both been rotated to be in the plane of the paper. The image of concentric circles for a range of $r$ values is shown in the $P^{\prime}$ plane.

This procedure can be performed with any pair of planes, but we loose no generality by restricting our attention to planes through the origin. Let $\mathbf{p}$ be any point on $S$ and define $F_{\mathbf{p}}=\Pi_{\mathbf{n}} \Pi_{\mathbf{p}}^{-1}$. We will identify but $P$ and $P^{\prime}$ with $R^{3}$ then any conformal mapping $H: R^{3}-\left\{\mathbf{q}_{1}\right\} \rightarrow R^{3}-\left\{\mathbf{q}_{2}\right\}$ has the form $H=F_{\mathbf{p}} \circ G$ where $G(\mathbf{x})=\alpha \mathbf{A x}+\mathbf{b}$ is a similarity. 
A general feature of conformal mappings of domains in Euclidean space is that they preserve round spheres and circles. Here planes are understood to be spheres of infinite radius and lines to be circles of infinite radius. A similarity of $R^{3}$ clearly preserves spheres and maps concentric spheres to concentric spheres. A conformal mapping which is not a similarity preserves spheres but will map concentric spheres to non concentric spheres (see Fig 2).

An example of the application of this mapping to a simple geometric figure is given in Figure 3.
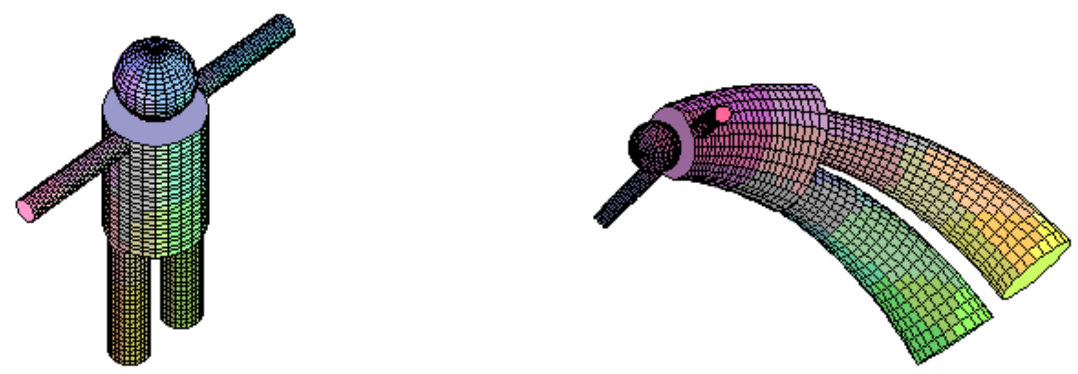

Figure 3. A simple 3 dimensional body and its image under the Möbius transformation $F$ defined in the text. Note that circles and spheres are preserved but that straight lines are generally taken to circles.

\section{Indentifiability of shape and conductivity}

We now see that the conductivity and the boundary configuration of an isotropic ohmic body can be determined by boundary measurement with an ambiguity of three parameters in the shape. To see this choose a configuration $\Omega_{1}$ and suppose that for this configuration a conductivity $\sigma_{1}$ can be found which is consistent with the complete measured data. Now let $\Omega_{2}$ be another such configuration with conductivity $\sigma_{2}$ consistent with the data. We may think of the first configuration and conductivity as being correct while the second configuration has the wrong shape and a consistent but wrong conductivity. We know from Section 3 that there must be a conformal mapping $F: \bar{\Omega}_{1} \rightarrow \bar{\Omega}_{2}$. This can be extended to a Möbius transformation defined on $R^{3}$ except possibly at a point in the domain and another in the codomain. Conversely any Möbius transformation $F$ defined on $\bar{\Omega}_{1}$ gives rise to a consistent configuration with $\Omega_{2}=F\left(\Omega_{1}\right)$. We see then that the ambiguity in the configuration has ten degrees of freedom, but seven of these arise from similarities so do not affect the shape. There are actually only three parameters we have to specify to fix the shape. A natural way to do this is to choose four independent (that is not coplanar) points $\mathbf{y}_{i} 0 \leq i \leq 3$ on 
$\partial M$. When these points are a embedded in $R^{3}, \mathbf{x}_{i}=\Psi\left(\mathbf{y}_{i}\right)$ they define a unique sphere with centre $\mathbf{c}$. We can express $\mathbf{c}$ in terms of these points as $\mathbf{c}=\mathbf{x}_{0}+\sum_{i=1}^{3} \lambda_{i}\left(\mathbf{x}_{i}-\mathbf{x}_{0}\right)$. The vector $\left(\lambda_{1}, \lambda_{2}, \lambda_{3}\right)$ gives the coordinates of $\mathbf{c}$ relative to the frame defined by the $\mathbf{x}_{i}$. Any similarity $G$ takes spheres to spheres preserving the centres thus $G(\mathbf{c})=G\left(\mathbf{x}_{0}\right)+\sum_{i=1}^{3} \lambda_{i}\left(G\left(\mathbf{x}_{i}\right)-G\left(\mathbf{x}_{0}\right)\right)$ and we see that the relative coordinates are preserved. Conversely any conformal mapping which is not a similarity will not preserve the centre of the sphere. The result can be summarised as follows:

\section{Theorem 5.1}

Once the relative coordinates of the centre of a sphere through four points on the boundary are given, an isotropic analytic conductivity and the shape of the boundary are determined by the boundary electrical data.

The argument above gives a heuristic proof of the theorem. For more rigorous, but technical proof one simply needs to check that $\left(\lambda_{1}, \lambda_{2}, \lambda_{3}\right)$ is a coordinate chart on the so called Möbius sphere which is the conformal group of the three sphere factored by the isotropy group of a point on the three sphere [8]. This isotropy group is isomorphic to the conformal group of $R^{3}$ so the Möbius sphere (which is diffeomorphic to the three sphere) is precisely conformal mappings (defined on $R^{3}$ except at a point) modulo Euclidean similarities.

While this result says that both the conductivity and the boundary shape can be determined by electrical data together with three spatial measurements, this is not to say that electrical measurement would be a sensible way to determine the boundary shape of a body with unknown conductivity. In medical imaging applications it would make sense to use mechanical or optical shape measurement.

Now consider the case where we have the boundary electrical data and attempt to recover the conductivity but we assumed the wrong shape. Except in the very unusual situation where the true shape and the assumed shape are related by a conformal transformation we will not be able to find a conductivity consistent with our measured data.

The good news for practical EIT is that, at least assuming the conductivity is isotropic, reconstruction algorithms should fail to converge if the shape is grossly wrong.

\section{Two dimensional problems}

Unfortunately many practical EIT systems perform their reconstructions using two dimensional models where the data is obtained from three dimensional bodies $[9,10,11]$. In this case the algorithms would not be expected to converge. To see this consider a three dimensional body with a smooth boundary and any reasonably behaved conductivity (differentiable for example). If a point source of current is applied to the boundary at a point $\mathbf{y}$ then the resulting potential will have a singularity of the form $1 /|\mathbf{x}-\mathbf{y}|$. If a two dimensional model is used the result of applying a point 
source of current will be a potential with a singularity of the form $\log |\mathbf{x}-\mathbf{y}|$. These asymptotic forms of the singularity in the potential even hold for anisotropic potentials. Details are given by Alessandrini [14].

Whatever conductivity, or indeed boundary shape, is chosen for the model this fundamental difference will remain. Provided sufficiently many electrodes are used and enough potential measurements made one will not be able to fit the two dimensional model to the three dimensional data.

Consequently a least squares minimisation algorithm will fail to fit the model to the conductivity beyond a certain error due to the wrong dimensional model. The error caused by the inaccurate boundary shape may be smaller and may not be noticed. In the case of the human chest a horizontal ring of electrodes is often used. These electrodes have non-zero height and one might reasonably expect these measurements to fit a to dimensional model better with taller electrodes For an account of the impact of shape errors on chest imaging see [12].

Where the data is obtained from a genuinely two dimensional phantom the existence of a conformal mapping between any two simply connected domains means that errors in boundary shape can be accounted for by distortions of the image. But this is an idealisation which assumes that $J$ is measured directly.

In the two dimensional case it may be reasonable to assume that that the electrode spacing is known but not their position. For example they may be positioned with the aid of a tape measure. In this case the shape and size are uniquely determined by boundary electrical data. To see this take the abstract manifold to be $M=\{z \in C:|z| \leq 1\}$ the closed unit disk in the complex plane (we assume here that the body is simply connected). Let $f: M \rightarrow C$ be a conformal mapping. Preserving electrode spacing corresponds, in the limit of numerous electrodes, to preserving the metric on the boundary. We see therefore that $\left|f^{\prime}(z)\right|=1$ for $|z|=1$. Now $\log f^{\prime}(z)$ is analytic for $|z|<1$ and $\operatorname{Re} \log f^{\prime}(z)=0$ for $|z|=1$. Hence $\log f^{\prime}(z)=\alpha i$ must be an imaginary constant and $f(z)=e^{i \alpha} z+c$ is just a rotation and translation. Another way in which a length scale may be fixed is to specify the current density $j$ (which has the dimensions Current/Length) rather than the current $J$.

We conclude that in two dimensions, once the arc length on the boundary is fixed no change in boundary shape can be accounted for by a change in conductivity and that both conductivity and boundary shape can be determined from the boundary electrical data.

Numerical experiments using two dimensional numerical phantoms, such as those reported by Jain [13] show that with the wrong boundary shape a regularised Newton's method will often produce an image with some correct conductivity features as well as some artefacts. However the boundary data from these images never fits the simulated data from a model with the correct boundary shape. Kiber et al [16] attempted to estimate the shape of the boundary from electrical data using a two dimensional model. They report reasonable success for an elliptical two dimensional tank of constant conductivity and some success using in vivo data from a human thorax. 


\section{Electrode Modelling}

Real EIT systems apply current and measure potential using a system of conductive electrodes applied to the surface of the body. Typically the size and shape of these electrodes is fixed, and the size is not negligible in comparison with the gaps between electrodes. Thus far we have adopted the convention of a continuum of electrode which allows us to apply any desired current over a given region of the boundary. Cheng et al [17] call this the 'no gap' model. A simple model which takes account of the size but not the high conductivity of the electrodes is the 'gap model'. In this case a constant current density is assumed on each electrode $E_{k} \subset \partial M k=1 \ldots K$

$$
\begin{aligned}
& \sigma \frac{\partial \phi}{\partial n}=I_{k} / \operatorname{Area}\left(E_{k}\right) \text { on each } E_{k} \\
& \sigma \frac{\partial \phi}{\partial n}=0 \text { off the electrodes. }
\end{aligned}
$$

Unlike the transcondcutance operator this type of condition is not invariant under change of coordinates on the boundary. Even if the shape and area of the electrodes was not fixed the condition of constant current density on the electrodes would not be preserved by a conformal mapping other than a similarity.

A more sophisticated model of electrodes, the 'shunt model' [17,18] takes account of the high conductivity of the electrode. In this case the potential is assumed constant on each electrode and the total current on each electrode is prescribed:

$$
\begin{aligned}
& \int_{E_{k}} J=I_{k} \text { and } \phi=V_{k} \text { (constant) for each electrode } E_{k}, \\
& \sigma \frac{\partial \phi}{\partial n}=0 \text { off the electrodes }
\end{aligned}
$$

If the shape of the electrodes were only known in the abstract coordinate system such conditions would be invariant under change of coordinates and so would allow the ambiguity of conformal maps as described above in both the two and three dimensional cases. However it is more realistic to assume a predetermined electrode shape and size in physical coordinates. The shape of circular but not square electrodes would be preserved under a general conformal mapping, but not their size.

\section{Acknowledgements}

I would like to thank Jack Lee, Simon Donaldson and Nairo Aparicio for helpful discussions on this work, and the Wellcome Trust for funding the experimental programme which inspires these theoretical developments.

[1] Metherall $\mathrm{P}$ et al., 3-Dimensional Electrical-Impedance Tomography, Nature, 1996, Vol.380, No.6574, pp.509-512 
[2] Barber DC and Brown BH eds, Physiol Meas, 1996, Vol.17, Suppl 4A.

[3] Beck M (ed) European Concerted Action on Process Tomography (ECAPT) 1994. Oporto Portugal, 24 - 26 March 1994

[4] Lionheart W.R.B., Conformal uniqueness results in anisotropic electrical impedance imaging, Inverse Problems 13, 1997, pp125-134

[5] Lee J.M. and Uhlmann G., "Determining Anisotropic Real-Analytic Conductivities by Boundary Measurements", Comm. Pure Appl. Math., vol 42, 1989, pp1097-1112.

[6] Sylvester, J., An Anisotropic Inverse Boundary Value Problem, Comm. Pure Appl Math,43, 1990, 201-232

[7] Kobayashi, S., Transformation Groups in Differential Geometry, Springer-Verlag, Berlin, 1972

[8] Kulkarni R.S and Pinkall U., Conformal Geometry, Vieweg, Braunschweig, 1988

[9] Smith RWM et al, A real-time Electrical-Impedance Tomography System for Clinical use - Design and Preliminary-Results. IEEE Trans Biomed Eng, 1995, Vol.42, No.2, pp.133-140

[10] Edic PM et al A Real-Time Electrical-Impedance Tomograph, IEEE Trans Biomed Eng, 1995, Vol.42, No.9, pp.849-859.

[11] Lionheart WRB et al, Electrical Impedance Tomography for high speed chest imaging, Physica Medica, Vol 13, Suppl 1, 1997

[12] Adler A et al. Impedance imaging of lung ventilation - do we need to account for chest expansion? IEEE Trans Biomed Eng, 1996, Vol.43, No.4, pp.414-420

[13] Jain H, Phd Thesis, Rensselaer Polytech Inst, NY, 1997

[14] Alessandrini G., Singular solutions of elliptic-equations and the determination of conductivity by boundary measurements, J. Diff. Eq., 84, 1990, pp. 252-272

[15] Nachman A.I., Global uniqueness for a two-dimensional inverse boundary value problem, Annals Math. 142, 1995, 71-96.

[16] Kiber M.A. Barber DC and Brown B.H, Estimation of Object Boundary Shape from the Voltage Gradient Measurements, Proc. Meeting on Electrical Impedance Tomography, Copenhagen, July 1990, Editor T.K. Hames, European Community Concerted Action on Electrical Impedance Tomogrphy.

[17] Cheng K-S, Isaacson D, Newell J.C, Gisser D.G, 'Electrode Models for Electric Current Computed Tomography', IEEE Trans Biomed Eng 36, 918-924, 1989.

[18] Somersalo E, Cheney M, Isaacson D, Existence and Uniqueness for electrode models for electric current computed tomography. Inverse Problems 52(4), 10231040, 1992 